\title{
Author Index for Volume 17
}

\begin{tabular}{|c|c|c|c|}
\hline Abdollahi, A. & 611 & Guo, X.Y. & 1,191 \\
\hline Ali, F. & 389 & Haghany, A. & 621 \\
\hline Azad, A. & 611 & Hassanzadeh, S.H. & 153 \\
\hline Bartolone, C. & 565 & Hiss, G. & 361 \\
\hline Başer, M. & 257 & $\mathrm{Hu}$, N.H. & 431 \\
\hline Baziar, M. & 621 & Huang, Q.H. & 337 \\
\hline Boudi, N. & 211 & Huh, C. & 283 \\
\hline Bovdi, V. & 17 & Jia, L. & 475 \\
\hline Carini, L. & 319 & Jow, S.-Y. & 415 \\
\hline Chen, H.Y. & 87 & Juriaans, S.O. & 223 \\
\hline Chen, J.L. & 337,447 & Klep, I. & 629 \\
\hline Chen, L.Y. & 525 & Külshammer, B. & 75 \\
\hline Chu, L.Z. & 699 & Kwak, T.K. & 257 \\
\hline Ciraulo, F. & 565 & Lee, C.I. & 283 \\
\hline Danz, S. & 75 & Lee, K.S.E. & 301 \\
\hline De Falco, M. & 203 & Lee, Y. & 283 \\
\hline De Filippis, V. & 319 & Li, A.H. & 173 \\
\hline de Giovanni, F. & 203 & $\mathrm{Li}, \mathrm{F}$. & 475 \\
\hline Di Vincenzo, O.M. & 319 & Li, J.B. & 181 \\
\hline $\mathrm{Du}, \mathrm{N}$. & 59 & Li, Q.S. & 173 \\
\hline Dudek, W.A. & 65 & Li, S.R. & 59 \\
\hline Endimioni, G. & 487 & Li, S.Z. & 101 \\
\hline Fang, J. & 595 & Li, Y.T. & 515 \\
\hline Fang, X.G. & 161 & Li, Y.Y. & 265 \\
\hline Fang, Y.J. & 375 & Lian, H.F. & 705 \\
\hline Feng, H.H. & 1 & Lin, W.Q. & 247 \\
\hline Feng, Y.Q. & 515 & Lin, Y.N. & 507 \\
\hline Geng, S.F. & 131 & Lin, Z.Q. & 507 \\
\hline Geng, Y.X. & 667 & Liu, D. & 431 \\
\hline Gong, S.-J. & 43 & Liu, Q.H. & 47 \\
\hline Guo, W.B. & 549 & Marcelo, A. & 425 \\
\hline
\end{tabular}


Massé, C. 27

Mendes-Gonçalves, S. $\quad 109$

Moghaddamfar, A.R. $\quad 121$

Mohammadi Hassanabadi, A. 611

Moori, J.

389

Moravec, P.

629

Muñoz Masqué, J.

425

Musella, C.

Niu, P.

203

161

Padmanabhan, R.

Peng, L.G.

Penner, $\mathrm{P}$.

Petrich, M.

$\mathrm{Qu}$, H.P.

Rejali, A.

Rodríguez Mielgo, C.

Rogério, J.R.

Saremi, H.

Shemetkov, L.A.

Shirmohammadi, N.

Shu, B.

Sica, C.

Skiba, A.N.

Sokolov, E.V.

Song, Q.W.

Srivastava, J.B.

$\mathrm{Su}$, J.H.

$\mathrm{Su}$, Y.C.

Sullivan, R.P.

Sun, S.H.

Sun, Y.L.

Tan, S.B.

Telloni, A.I.

Tiep, P.H.

Trokhimenko, V.S.

Vedadi, M.R.
Vorob'ev, N.N.

557

Wang, H.

27

Wang, J.

161

Wang, Q.W.

345

Wang, S.

365

Wang, S.H.

685

Wang, Y.

295

Wang, Y.D.

659

Wang, Y.M.

495

Wei, H.Q.

495

Willems, W.

541

Wismath, S.L.

27

$\mathrm{Wu}$, D.J.

659

Wu, Y.N.

247

Xia, Z.S.

705

Xie, W.

345

Xin, B.

181

241

469

$\mathrm{Xu}, \mathrm{M} . \mathrm{C}$.

525

$\mathrm{Xu}, \mathrm{X} . \mathrm{N}$.

495

447

Yao, L.L.

583

101

43

345

153

611

101

525

11

685

365

121 